ARTICLE

Received 24 Sep 2015 | Accepted 9 Dec 2015 | Published 27 Jan $2016 \quad$ DOl: 10.1038/ncomms10426 OPEN

\title{
Misorientation-angle-dependent electrical transport across molybdenum disulfide grain boundaries
}

Thuc Hue Ly ${ }^{1, \star}$, David J. Perello ${ }^{1, \star}$, Jiong Zhao ${ }^{1, \star}$, Qingming Deng ${ }^{2}$, Hyun Kim ${ }^{1,3}$, Gang Hee Han ${ }^{1}$, Sang Hoon Chae $^{1}$, Hye Yun Jeong ${ }^{1,3} \&$ Young Hee Lee ${ }^{1,3}$

Grain boundaries in monolayer transition metal dichalcogenides have unique atomic defect structures and band dispersion relations that depend on the inter-domain misorientation angle. Here, we explore misorientation angle-dependent electrical transport at grain boundaries in monolayer $\mathrm{MoS}_{2}$ by correlating the atomic defect structures of measured devices analysed with transmission electron microscopy and first-principles calculations. Transmission electron microscopy indicates that grain boundaries are primarily composed of 5-7 dislocation cores with periodicity and additional complex defects formed at high angles, obeying the classical low-angle theory for angles $<22^{\circ}$. The inter-domain mobility is minimized for angles $<9^{\circ}$ and increases nonlinearly by two orders of magnitude before saturating at $\sim 16 \mathrm{~cm}^{2} V^{-1} \mathrm{~s}^{-1}$ around misorientation angle $\approx 20^{\circ}$. This trend is explained via grain-boundary electrostatic barriers estimated from density functional calculations and experimental tunnelling barrier heights, which are $\approx 0.5 \mathrm{eV}$ at low angles and $\approx 0.15 \mathrm{eV}$ at high angles $\left(\geq 20^{\circ}\right)$.

\footnotetext{
${ }^{1}$ IBS Center for Integrated Nanostructure Physics (CINAP), Institute for Basic Science, Sungkyunkwan University, Suwon 440-746, Korea. ${ }^{2}$ IFW Dresden, Institute of Solid State Research, P.O. Box 270116, D-01171 Dresden, Germany. ${ }^{3}$ Department of Energy Science, Sungkyunkwan University,

Suwon 440-746, Korea. ${ }^{\star}$ These authors contributed equally to this work. Correspondence and requests for materials should be addressed to

Y.H.L. (email: leeyoung@skku.edu).
} 
U nderstanding the atomic structures of grain boundaries (GBs) is essential to controlling and improving electrical transport properties in both bulk and low-dimensional materials. In traditional mesoscopic semiconductors, GBs are scattering sites for majority carriers and degrade transport via the formation of electrostatic barriers ${ }^{1,2}$. Such degradation is amplified in low-dimensional materials due to the limited phase space and reduction in Coulomb screening ${ }^{3}$. Two-dimensional (2D) transition metal dichalcogenides are one class of lowdimensional materials with unique electrical and electro-optical properties showing promise for emerging valleytronics ${ }^{4}$ and soft electronics applications ${ }^{5}$. However, large-area monolayer transition metal dichalcogenides synthesized via chemical vapour deposition (CVD) inevitably possess GBs between randomly oriented crystal domains. Early theoretical research involving density functional theory (DFT) calculations for $\mathrm{MoS}_{2}$ predicted the possibility of both metal and semiconductor GB characteristics, depending on the local atomic structures ${ }^{6-8}$. However, experimental assessment of $\mathrm{MoS}_{2}$ GB transport is still under debate in the literature ${ }^{6-11}$ due to a large device-to-device performance variation, poor single-domain carrier mobility, and, most importantly, lack of correlation between transport properties and GB atomic structures.

Here, we overcome these difficulties by directly correlating four-probe transport measurements across single GBs with both high-resolution transmission electron microscopy (TEM) imaging of the measured devices and first-principles calculations. Using an inert-environment fabrication procedure, we observe a positive and nonlinear correlation between field effect mobility $\left(\mu_{\mathrm{FE}}\right)$ and misorientation angle $(\alpha)$. The precise boundary location, orientation and atomic structure of transistor devices were also examined by TEM, results of which were crossreferenced with further characterization and imaging of pristine $\mathrm{MoS}_{2}$ GBs. On using DFT to calculate the $\alpha$-dependent local GB band structures, equilibrium electrostatic GB barriers were found to be correlated with experimentally determined barriers, which gives a clear understanding of the underlying mobility dependences.

\section{Results}

Electrical characterization. Monolayer $\mathrm{MoS}_{2}$ flakes (typical sizes of $20-40 \mu \mathrm{m}$ ) were grown by CVD and subsequently transferred onto $\mathrm{SiO}_{2}(100 \mathrm{~nm}) / \mathrm{Si}$ substrates (see Methods). GB positions were then initially determined by dark-field optical microscopy images while considering the growth kinetics (Supplementary Fig. 1). Regions with no sign of wrinkling or multilayers were then selected to prevent misinterpretation of the transport mechanism. To suppress interaction with oxygen-related functional groups, further device fabrication was performed in a glove box $\left(\mathrm{H}_{2} \mathrm{O}, \mathrm{O}_{2}<1\right.$ p.p.m. $)$ and only acetone solvent was used during the electron-beam lithography procedures to minimize additional GB functionalization.

$V_{\mathrm{gs}}$-dependent transport measurements were performed on an elongated Hall-bar geometry to allow single-crystal and GB four-probe sheet resistance $\left(R_{\mathrm{s}}\right)$ and $\mu_{\mathrm{FE}}$ comparison. Figure 1a shows the schematic of a typical carrier transport across a GB, while a representative multi-terminal device tested in this work is displayed in Fig. 1b. A GB with an optical microscopydetermined misorientation angle of $19^{\circ}$ is located near the centre of the monolayer $\mathrm{MoS}_{2}$ film (red-dashed line), where the white dashed lines (inset) denote the pre-etch shape of $\mathrm{MoS}_{2}$ flakes (Supplementary Fig. 2). The $R_{\mathrm{s}}$ and $\mu_{\mathrm{FE}}$ associated with intradomain and inter-domain transport were measured as a function of $V_{\mathrm{gs}}$ by choosing appropriate electrodes either within the domain or across the GB line, respectively (Fig. 1c and
Supplementary Fig. 7a). The selected-area electron-diffraction (SAED) pattern (right inset of Supplementary Fig. 3b) and scanning (STEM) findings of the exact device in Fig. $1 \mathrm{~b}$ both revealed that $\alpha=19^{\circ}$, which further agrees with the optical estimates within a statistical error of $\pm 2^{\circ}$ (Supplementary Fig. 3). In this $19^{\circ}$ sample, the intra-domain (inter-domain) $\mu_{\mathrm{FE}}=24-60$ (16) $\mathrm{cm}^{2} \mathrm{~V}^{-1} \mathrm{~s}^{-1}$ and $R_{\mathrm{s}}=30-85$ (95) $\mathrm{k} \Omega \mathrm{sq}^{-1}$. After completing the electrical measurements, the devices were expeditiously transferred to an Au TEM grid (an Au grid was used to suppress additional oxidation of the sample as the moisture level should be minimized to prevent sample damage). STEM analysis of the boundary revealed that the GB line consists of a series of 5-7 membered ring cores, as indicated by the orange-dashed line in the inset of Fig. 1c. (Mo atoms are denoted in blue, while $\mathrm{S}$ atoms are displayed in yellow.)

For further angle-dependent transport analysis, a statistical distribution of the mobility was obtained from 43 devices (29 intra-domain and 14 inter-domain, refer to Supplementary Fig. 3 for raw mobility data), as shown in Fig. 1d. A decisive cutoff separates intra-domain and inter-domain data at $\mu_{\mathrm{FE}}=16$ $\mathrm{cm}^{2} \mathrm{~V}^{-1} \mathrm{~s}^{-1}$. The intra-domain $\mu_{\mathrm{FE}}$ dataset forms an ordered but highly dispersive statistical distribution with a minimum (maximum) of $\mu_{\mathrm{FE}}=17 \quad(115) \mathrm{cm}^{2} \mathrm{~V}^{-1} \mathrm{~s}-1$, which results from conduction band-tail states localized near the conduction band edge $^{12}$ (Supplementary Fig. 5). Figure 1d shows the raw data and natural-log of $\mu_{\mathrm{FE}}$ histograms (inset); excellent fitting to a log-normal distribution was observed, with an expected $\mu_{\mathrm{FE}}=44 \mathrm{~cm}^{2} \mathrm{~V}^{-1} \mathrm{~s}^{-1}$. Notably, seven devices had a $\mu_{\mathrm{FE}}>75 \mathrm{~cm}^{2} \mathrm{~V}^{-1} \mathrm{~s}^{-1}$ and on-state $R_{\mathrm{s}}$ was consistent with the best-reported exfoliated results ${ }^{13}$ (also see Supplementary Fig. 6).

With statistically reliable intra-domain mobility data to contrast, the inter-domain misorientation angle-dependent mobility was measured. Results are shown in Fig. 1e $\left(R_{\mathrm{s}}\right.$ data are presented in Supplementary Fig. 7). Interestingly, the interdomain mobility increases for small angles $\left(8-20^{\circ}\right)$, although some variability exists in the low-angle data. Above $20^{\circ}, \mu_{\mathrm{FE}}$ saturates below the $16 \mathrm{~cm}^{2} \mathrm{~V}^{-1} \mathrm{~s}^{-1}$ intra-domain cutoff. Overall, the inter-domain mobilities vary by 2 orders of magnitude across the tested range of $\alpha$. It was further observed that although devices with $8-20^{\circ}$ inter-domain misorientation display poor transport, these samples consistently conduct, as demonstrated by the large number of inter-domain data points within this range. Conversely, samples within the $20-60^{\circ}$ range show better transport properties; many GBs were insulating (broken) due to a torn/poor interface. One example is the $34^{\circ}$ sample (Fig. 1e), which was measurable only after annealing at $420 \mathrm{~K}$, and then quickly became insulating and unmeasurable at $300 \mathrm{~K}$. Other missing inter-domain data of Fig. 1e resulted from similar insulating behavior, likely due to tearing during substrate transfer (Supplementary Fig. 8). This observation agrees with previous experiments on 3D crystals, where high-angle samples frequently shattered after growth ${ }^{14}$. The peculiarly high mobility of the inter-domain $19^{\circ}$ device results from the boundary crossing the corner of the contact (Fig. 1b,c), which was discovered during post-transfer scanning electron microscopy or STEM analysis.

Structural characterization. To directly correlate the transport results to the atomic defect structures, we performed extensive TEM/STEM analysis of the aforementioned devices and many other representative samples; the results are summarized in Fig. 2. The diffraction contrast in the dark field (DF)-TEM images (Figs 2a-d) clearly reveals the morphology and location of the GBs. Low angle (LA)-GBs $\left(0-13^{\circ}\right)$ are rather straight, and formed by aligned dislocation cores, visible as dotted lines in the DF images (Fig. 2a). The SAED is presented in the inset of Fig. 2a, 

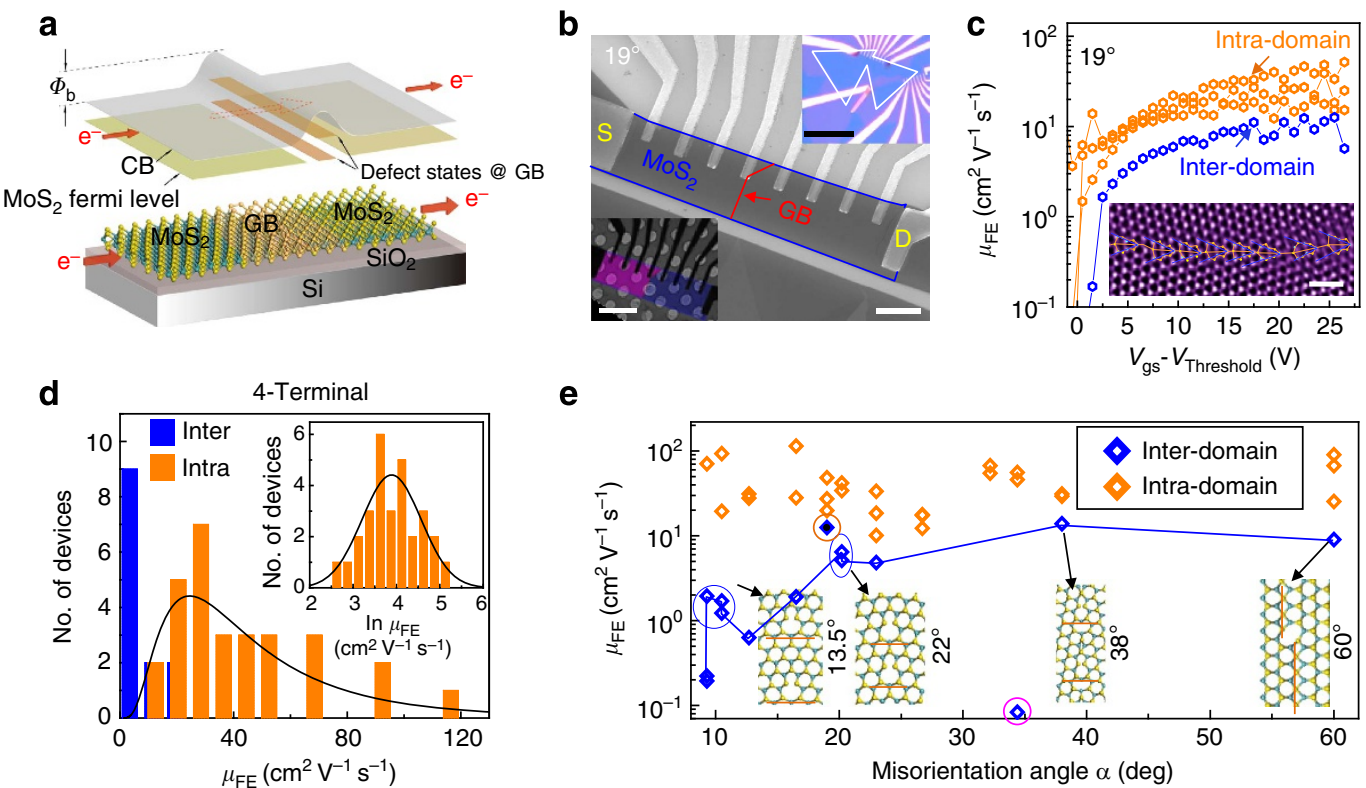

\begin{abstract}
Figure 1 | Measurements and statistics on Intra-domain vs inter-domain transport. (a) Experimental schematic of carrier transport across a grain boundary. (b) SEM image of a device sample on $\mathrm{SiO}_{2} / \mathrm{Si}$ wafer; scale bar, $3 \mu \mathrm{m}$. After device characterization, the device was transferred to a TEM grid; scale bar, $6 \mu \mathrm{m}$ (left inset). Right inset: Optical micrograph of the device with pre-etch flake shape outlined; scale bar, $30 \mu \mathrm{m}$. (c) Electrical performance of the device in panel (b) with $\alpha=19^{\circ}$. Intra-domain regions always have a higher $\mu_{\mathrm{FE}}$ and reduced $R_{\mathrm{S}}$ when compared to inter-domain. An STEM image of the boundary is shown in the inset. Inset scale bar, $1 \mathrm{~nm}$. (d) Statistical distribution of the intra- and inter-domain mobilities with $\mu_{\mathrm{FE}(\text { inter) }}<16 \mathrm{~cm}^{2} \mathrm{~V}^{-1} \mathrm{~s}^{-1}<\mu_{\mathrm{FE}(\text { intra). }}$ The intra-domain $\mu_{\mathrm{FE}}$ displays a log-normal distribution (black line) with expectation value $\mu_{\mathrm{FE}}=44 \mathrm{~cm}^{2} \mathrm{~V}^{-1} \mathrm{~s}^{-1}$, as exemplified by $\ln \left(\mu_{\mathrm{FE}}\right)$ in the inset. (e) The $\alpha$-dependent $\mu_{\mathrm{FE}(\text { inter) }}$ (blue) for 10 different angles with $\mu_{\mathrm{FE}(\text { intra) }}$ (total of 43 devices). Here, $\mu_{\mathrm{FE}(\text { inter) }}$ increases with a rise in $\alpha$ from $9^{\circ}$ to $20^{\circ}$, but saturates for $\alpha>20^{\circ}$ at $\mu_{\mathrm{FE}(\text { inter })}<16 \mathrm{~cm}^{2} \mathrm{~V}^{-1} \mathrm{~s}^{-1}$. The $19^{\circ}$ dislocation is of the 'Down'-type, while the others are 'Up'-type.
\end{abstract}

while the high-resolution STEM annular dark field (ADF) image is shown in Fig. 2e.

In bulk specimens, LA-GBs are composed of periodically piled edge dislocations. The distance between the edge dislocation cores can be estimated by $d=\mathbf{b} / \sin (\alpha)$, where $\mathbf{b}$ is the Burgers vector. The dislocations are composed of 'Up' or 'Down' types (Figs $2 \mathrm{j}-\mathrm{m}$ ) that correspond to S-S bonding or Mo-Mo bonding at the core, respectively. Due to the three-fold (not six-fold) symmetry of the $\mathrm{MoS}_{2}$ monolayer, a lack of inversion symmetry exists. Such a scenario induces the formation of two types of LA-GBs with the aforementioned 'Up' or 'Down' dislocations along the armchair directions, as highlighted in Fig. 2n. All devices investigated for electrical transport were of the 'Up'-type boundary variety (except for $19^{\circ}$, which is not utilized in the comparison) and thus comparison can be made. Furthermore, the energies associated with 'Up/Down' dislocations are similar, and both are energetically favourable since they both have the smallest Burgers vector [11-20]. Due to kinetic growth limitations, GBs with overall relative orientation between the armchair directions of $\mathrm{MoS}_{2}$ are also composed of $[11-20]$ dislocations ('Up/Down'). The boundary is decomposed into short armchair-oriented sections and is slightly kinked on the mesoscopic scale (Fig. 2a). For bulk materials, LA-GBs usually imply $\alpha<6^{\circ}$ due to strong interactions between the strain fields generated by adjacent dislocation cores at higher angles ${ }^{15}$. However, basal plane strain relaxation can occur in $2 \mathrm{D}$ materials via local rippling or buckling ${ }^{16}$, thereby allowing for an extension of LA-GB theory to larger angles. The periodicity of the dislocation core distance is inversely proportional to $\alpha$, in accordance with the classical LA-GB theory (Fig. $2 \mathrm{i}$ and Supplementary Fig. 9), but the range greatly exceeds the classical limit and is extended up to $13^{\circ}$, at which point the GB lines are bent by intense strain fields. Thermodynamically, these LA-GBs should follow a straight line in a direction perpendicular to the Burgers vector (armchair directions in $\mathrm{MoS}_{2}$ ), which is consistent with our observations (Supplementary Fig. 10).

High-angle (HA)-GBs between two domains can be classified as either coherent or incoherent, the former of which refers to GBs that possess a periodic lattice. Using notation from coincident site lattice theory ${ }^{17}$, the most favourable coherent boundaries for monolayer $\mathrm{MoS}_{2}$ are $\Sigma 7\left(22^{\circ}\right), \Sigma 13\left(28^{\circ}\right), \Sigma 19$ $\left(13^{\circ}\right)$, and so on (Supplementary Fig. 11). From the results of TEM/STEM characterizations and DFT calculations (Supplementary Fig. 12), we observed that most HA-coherent GBs also follow straight lines composed of continuous 5-7 ring cores, in a manner similar to LA-GBs (Figs $2 \mathrm{c}, \mathrm{g}$ ). Any GB with an $\alpha$ that deviates from coherent angles will be an incoherent GB (Fig. 2b). A curved (non-straight) GB can be identified in the atomic scale images (Fig. 2f). When compared to coherent GBs, the incoherent GBs have a larger aperiodic width of defects, whereas the mirror GBs $\left(60^{\circ} \mathrm{GB}\right)$ are mostly straight along the zigzag direction and composed of a series of 4-4 cores with few kinks (Figs 2d,h). However, when the mirror GB follows the armchair direction (due to growth kinetics), it is decomposed into a series of 4-8 cores (Supplementary Fig. 13).

In terms of mechanical stability, LA GBs are mostly stable, regardless of the GB type. This explains how we were able to accumulate a large set of data points, as shown in Fig. 1e. However, in the HA region, only coherent GBs with $\alpha=22^{\circ}, 38^{\circ}$ and $60^{\circ}$ were stable. While many samples contained boundaries with intermediate angles, transport data were not collected for these specimens because GBs were easily fragmented during the transfer process. Since incoherent GBs are generally wider with a complex array of defect structures (see Fig. 2f), they are 
a

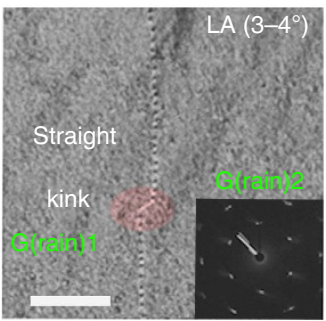

e

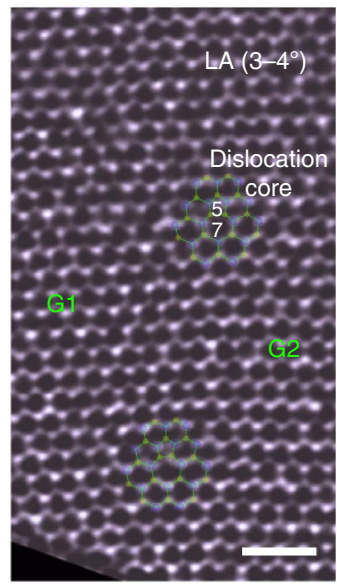

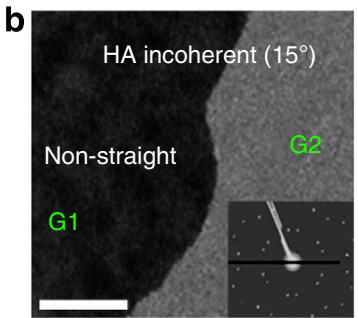

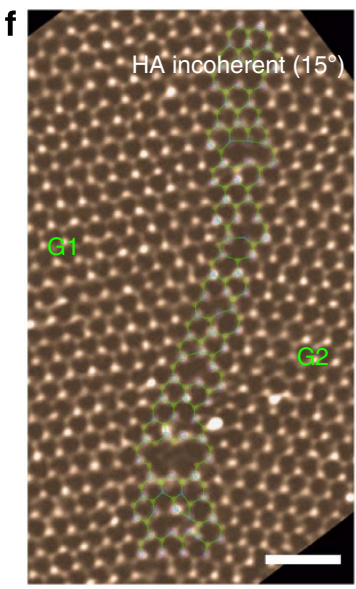

c

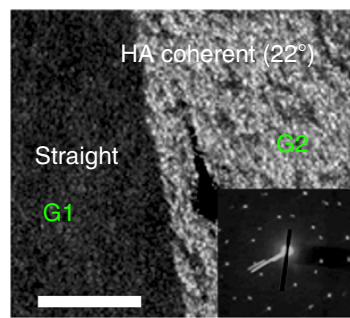

$\mathbf{g}$

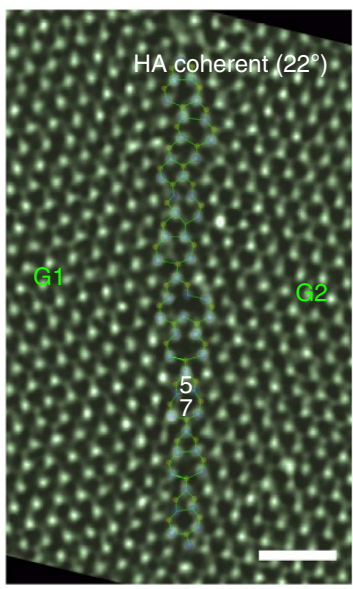

d

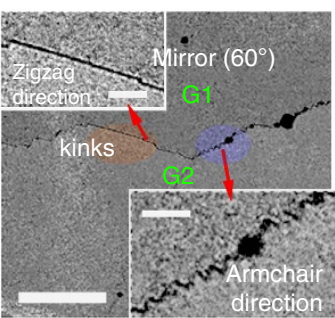

h

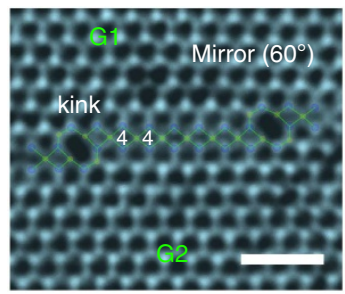

i

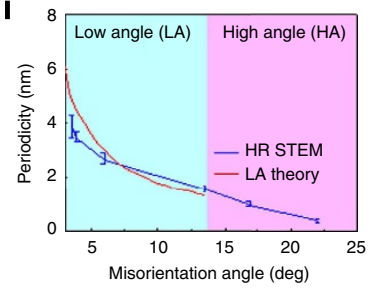

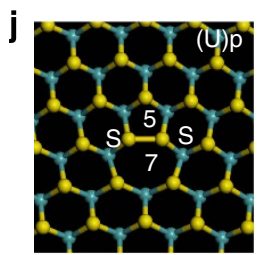
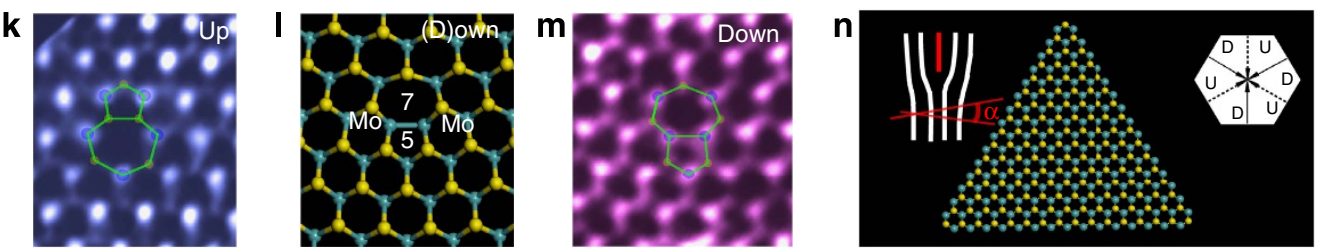

Figure 2 | TEM characterization of the $\mathbf{M o S}_{\mathbf{2}} \mathbf{G B s}$ showing misorientation angle dependence. (a-d) DF-TEM images of low-angle (3-4 ${ }^{\circ}$, high-angle incoherent $\left(15^{\circ}\right) /$ coherent $\left(22^{\circ}\right)$ and mirror GBs $\left(60^{\circ}\right)$; scale bar, $50 \mathrm{~nm}$. The insets in (a-c) show SAED patterns of the GBs, while the two inset images in (d) display zigzag (upper part) and armchair direction (lower part) mirror GBs. The scale bars in the latter two insets are $20 \mathrm{~nm}$. (e-h) High-resolution ADF-STEM images correlated to the four cases presented in (a-d); false colour was applied. Scale bar, $1 \mathrm{~nm}$. The dislocation cores and GBs were superimposed with atomistic models; blue and yellow circles denote Mo and S atoms, respectively, while the two domains are marked as $\mathrm{G} 1$ and $\mathrm{G} 2$. (i) Experimentally measured periodicity of the dislocation cores vs. misorientation angle with LA theory fitting. (j-m) Two types of LA-GBs: 'Up' type (S-S bond) and 'Down' type (Mo-Mo bond) (5-7) dislocation cores. The DFT-optimized models for each dislocation type are shown in (j,I), while ADF images superimposed with atomistic models are displayed in $(\mathbf{k}, \mathbf{m})$. (n) Atomistic model for a triangular flake with Mo-terminated zigzag edges, the upper left inset shows the 'Up' type dislocation scheme, while the upper right inset shows the six armchair directions in $\mathrm{MoS}_{2}$ with either 'Up' or 'Down' type dislocations. The directions of all schemes are correlated and aligned.

energetically less stable when compared to coherent GBs. This agrees well with the theoretical prediction that the total energy usually increases in response to the misorientation angle ${ }^{18}$.

\section{Discussion}

Dislocations and GBs in III-V systems (e.g., GaN) have previously been modeled by a generalized line charge with density $\rho_{\mathrm{L}} \propto$ defect density. Increasing $\rho_{\mathrm{L}}$ induces a larger electrostatic potential and results in a lower effective mobility ${ }^{19,20}$. In comparison, here we observe a positive and nonlinear correlation between $\mu_{\mathrm{FE}}$, the dislocation core density and $\alpha$. To explain why greater dislocation core density permits greater inter-domain mobility, and if results are consistent with the aforementioned theoretical models, we first consider whether the boundary is charged. $I_{\mathrm{ds}}-V_{\mathrm{gs}}$ curves from 26 two-terminal FETs (Fig. 3a) reveal that $I_{\text {off }}$ is rather invariant and limited by leakage current, while hysteresis is trivial and a uniform $0.25 \mathrm{~V}(300 \mathrm{~K})$, equal to the $V_{\mathrm{gs}}$ point spacing. Boundary charge must therefore be fixed, which is further examined by comparing respective $V_{\text {th }}$ values. As transport is band-tail limited and a well-defined saturation regime is absent in the $I_{\mathrm{sd}}-V_{\mathrm{gs}}$ curves (Fig. 3a), $V_{\text {th }}$ is defined by the $V_{\mathrm{gs}}$ logarithmic turn-on point corresponding to the peak subthreshold swing (SS). This point is denoted at $V_{\mathrm{gs}}-V_{\mathrm{th}}=0$ by a dotted line in Fig. 3a. We found $-11 \mathrm{~V}<V_{\text {th }}$ $<-5 \mathrm{~V}$ for all samples (Fig. 3b), which is comparable to that obtained from STM studies of atomically clean exfoliated samples ${ }^{21}$. A positive $1.6-\mathrm{V}$ shift (in median value) is observed in the interdomain samples compared with intra-domain, which is clear evidence for the existence of an electrostatic potential barrier. This potential barrier can be estimated by noting that, at $V_{\text {th }}, \mathrm{SS} \sim 230 \mathrm{mV} \mathrm{dec}^{-1}$. Since peak SS $=\ln (10) k T \times\left[1+\left(C_{\mathrm{MoS} 2}+C_{\mathrm{loc}}\right) / C_{\mathrm{SiO} 2}\right]$ is measured at the Fermi level $\left(E_{\mathrm{F}}\right)$ within the bandgap, $C_{\mathrm{MoS}_{2}} \approx 0$. Substituting $C_{\mathrm{SiO}_{2}}$ and SS above, we observe that $C_{\mathrm{loc}}=76 \mathrm{nF} \mathrm{cm}^{-2}$. An order-ofmagnitude estimate for the potential barrier height $\Phi_{\mathrm{b}}$ is obtained from an equivalent circuit model with $\mathrm{SiO}_{2}$ capacitance in series with the $\mathrm{MoS}_{2}$ self-capacitance, $\Delta E_{\mathrm{F}}=\Delta V_{\mathrm{th}}\left(C_{\mathrm{SiO} 2} / C_{\mathrm{loc}}\right)=190 \mathrm{meV}$ (see Methods).

However, in accordance with $\mu_{\mathrm{FE}}$, inter-domain $I_{\mathrm{on}} / I_{\mathrm{off}}$, and $R_{\mathrm{S}}$ results (Figs 3a and 1), $\Phi_{\mathrm{b}}$ is $\alpha$-dependent. For $9^{\circ} \leq \alpha \leq 60^{\circ}$ the core-density periodicity is less than $2 \mathrm{~nm}$, and thus electrostatic 

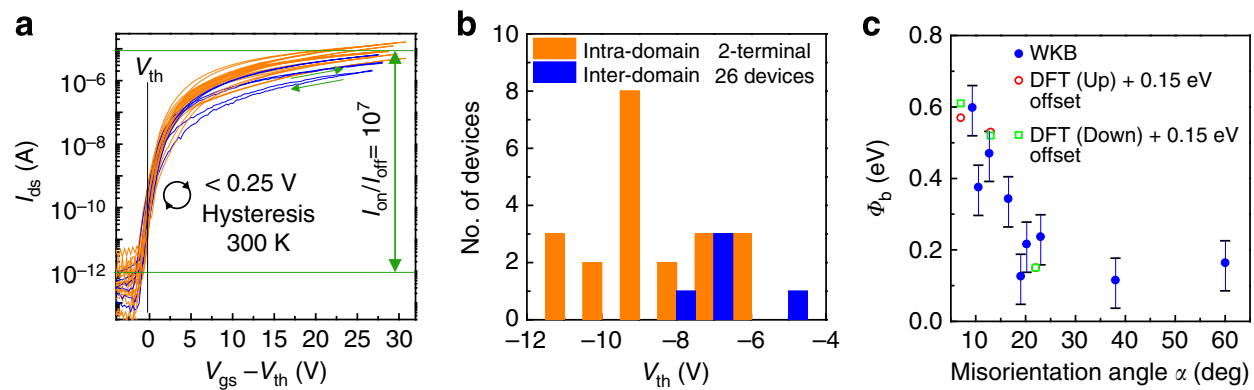

Figure 3 | Extraction of Schottky barrier height from the threshold voltage. (a) Dual sweep $I_{\mathrm{ds}}-V_{\mathrm{gs}}$ data for 26 different intra-/inter-domain two-terminal FETs at $300 \mathrm{~K}$. Consistent and trivial $0.25-\mathrm{V}$ clockwise hysteresis was observed for all 26 devices, regardless of the inter-/intra-domain status. Intra-domain devices have an average $I_{\text {on }} / I_{\text {off }}=10^{7}$ at $300 \mathrm{~K}$, while the inter-domain $I_{\text {on }} / I_{\text {off }}$ was reduced and misorientation angle-dependent. (b) Distribution of $V_{\text {th }}$ from panel (a) with a bin size of $0.25 \mathrm{~V}$. The median of the inter-domain $V_{\text {th }}$ was shifted by $+1.6 \mathrm{~V}$ from that of the intra-domain. (c) Inter-domain tunneling barrier height $\Phi_{\mathrm{b}}$ calculated via JWKB approximation when assuming a parabolic barrier shape and a transmission probability $T_{\mathrm{gb}}=\mu_{\mathrm{FE}(\mathrm{inter})} /$ $\mu_{\mathrm{FE}(i n t r a)}$. The error bars are based on one standard deviation of the intra-domain $\mu_{\mathrm{FE}}$.

perturbation is essentially a 1D barrier. The $\alpha$-dependence of the effective $\Phi_{\mathrm{b}}$ is calculated via the Jeffreys-WentzelKramers-Brillouin (JWKB) approximation ${ }^{22}$ with an on-state transmission probability $(T)$ based on the experimental $\mu_{\mathrm{FE}(\text { inter })}=T_{\mathrm{GB}} \mu_{\mathrm{FE}(\text { intra) }}$. Assuming a parabolic-shaped potential with a peak magnitude $\Phi_{\mathrm{b}}$ and $8 \mathrm{~nm}$ width based on STM observations $^{23}$, the JWKB approximation gives $T_{\mathrm{GB}}=$ $\exp \left(-8.7 \Phi_{\mathrm{b}}\right)$. Fitting to experimental data, it was found that $\Phi_{\mathrm{b}}$ peaks above $0.5 \mathrm{eV}$ for $\alpha<10^{\circ}$ and then decreases to a saturation point of $\sim 0.15 \mathrm{eV}$ for $\alpha>20^{\circ}$ (Fig. 3c). The error bounds are based on one standard deviation of the intra-domain log-normal distribution (Fig. 1d).

Finally, DFT was used to calculate the band structures of various GBs based on atomic geometries observed in TEM (Fig. 4). Representative coherent GBs of $7^{\circ}, 13^{\circ}$ and $22^{\circ}$ were selected (Fig. 4c). The dislocation 5-7 ring core structures for these angles are similar, but a larger bond length variation occurs in the vicinity of dislocation cores for smaller $\alpha$ was observed (Supplementary Figs 14,15). Calculations indicate the bands near the Fermi level (long-dashed red line) are almost flat with negligible dispersion for all three angles, indicating strong Fermi level pinning. The first two bands above the Fermi level (red line) come mostly from the Mo $4 \mathrm{~d}$ orbital at the 5-7 dislocation cores ('Down' core for the first band and 'Up' core for the second band). These energy levels near the Fermi level may serve as resonant charge-scattering centres, a notion that can be similarly surmised from the strongly localized charges at the GBs (Fig. 4c, 'Down' core at the centre and 'Up' core at the right edge of the supercell). In contrast, at the mirror boundary, the Fermi level crosses the completely delocalized state in the middle of $(\Gamma-\mathrm{Y})$, revealing metallic character due to the 4-4 ring dislocation cores (Supplementary Fig. 16). The delocalized metallic feature with weak pinning provides a likely explanation for the high mobility of the mirror GBs.

Angle-dependent transport was then modelled by considering local band bending at the boundary induced during charge equilibration, as shown schematically in Fig. $4 \mathrm{~d}$. At equilibrium, the interfacial $\Phi_{\mathrm{b}}$ is approximated by $\Phi_{\mathrm{b}}=W_{\mathrm{GB}}-W_{\mathrm{MoS} 2}-E_{\mathrm{d}}$, where $W_{\mathrm{GB}}=E_{\mathrm{c}}+E_{\mathrm{F}}$ is the $\mathrm{GB}$ work function, $W_{\mathrm{MoS} 2}$ is the $\mathrm{MoS}_{2}$ work function and $E_{\mathrm{d}}$ is the trap energy with respect to the GB work function. $E_{\mathrm{d}}$ is fixed due to Fermi level pinning, resulting in the equilibrium band diagram displayed at the bottom of Fig. $4 \mathrm{~d}$. The work functions for the three GB structures obtained by DFT are $5.67 \mathrm{eV}\left(7^{\circ}\right), 5.71 \mathrm{eV}\left(13^{\circ}\right)$ and $5.50 \mathrm{eV}$ $\left(22^{\circ}\right)$. Due to the presence of band-tail dispersion in the pristine $\mathrm{MoS}_{2}, W_{\mathrm{MoS} 2}$ is unknown and $V_{\mathrm{gs}}$-dependent, and an exact $\Phi_{\mathrm{b}}$ is undefined. However, relative values of $\Phi_{\mathrm{b}}$ are compared with the JWKB experimental results in Fig. 3c; an offset of $150 \mathrm{meV}$ was added to the calculated values. Both the 'Up' and 'Down' dislocation types show nearly identical trend, with $\Phi_{\mathrm{b}}$ decreasing at higher $\alpha$. The localization of charges at the boundary and subsequent Fermi level pinning determine the effective GB potential barrier height. GBs with defect states that are energetically farther from the conduction band (smaller $E_{\mathrm{d}}$ ) will have greater $\Phi_{\mathrm{b}}$ values. Consequently, in $\mathrm{MoS}_{2}$ the exact GB atomic structure determines the barrier, and, although the density of dislocation cores increases with $\alpha$, the charge density per unit length actually decreases as dislocation density increases. These results indicate that boundary transport is consistent with the previous theoretical treatment of $2 \mathrm{D}$ systems, although no exploration of the exact scattering mechanism at the GB is possible here.

For incoherent HA-GBs, more complicated defects than 5-7 cores exist, along with a greater number of dangling bonds in a zigzag shape or containing holes (Fig. 2), which cannot be explained by our JWKB tunnelling barrier treatment. As opposed to a straight potential barrier found in coherent GBs, a nonperiodic kinked boundary likely forms a wider depletion region with regions of greater localized charge density. Furthermore, due to growth limitations for LA-GBs $\left(<7^{\circ}\right)$, we were unable to characterize the transport properties for very small $\alpha$. In this regime, the induced potential perturbations from individual dislocation cores will become more isolated as $\alpha$ decreases. We postulate that carriers will simply pass within the sawtooth-like GB potential, leading to improved mobility with reduced $\alpha$.

In conclusion, we have provided a more unified picture of the relationship between mobility, merging angle and atomistic structures of the GBs of monolayer $\mathrm{MoS}_{2}$. The results provide practical expectations regarding transport properties in large-area films, which will be restricted largely by the poor mobility across LA GBs. The results obtained in this work are applicable to other similar $2 \mathrm{D}$ systems, and contribute to the fundamental understanding of transport in semiconductors.

\section{Methods}

$\mathbf{M o S}_{\mathbf{2}}$ grown on $\mathrm{SiO}_{2} / \mathrm{Si}$ wafer by CVD. OptiPrep density gradient medium (Sigma-Aldrich, D1556, 60\% (w/v) solution of iodixanol in water) (defined as solution A) was purchased and used as a medium to dissolve promoter and precursor. It has no influence on growth. The promoter was prepared by dissolving sodium cholate (SC) hydrate (Sigma-Aldrich, C6445) in deionized (DI) water $(0.6 \mathrm{~g}$ of SC in $30 \mathrm{ml}$ of DI water), defined as solution B. A solution-phase ammonium heptamolybdate (AHM) precursor was also prepared by dissolving ammonium molybdate tetrahydrate (Sigma-Aldrich, 431346) into DI water (defined as solution 

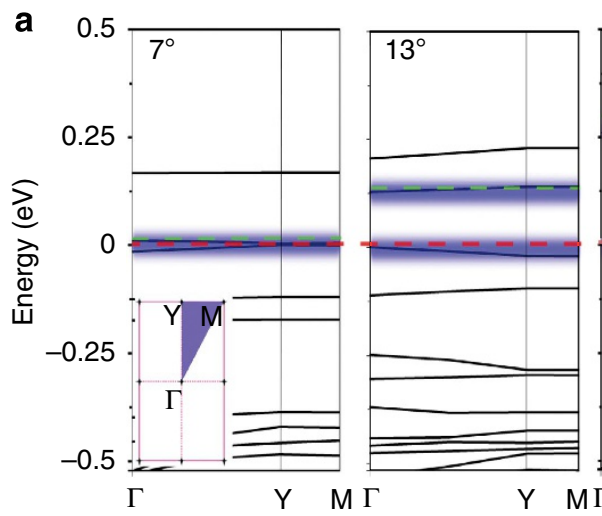

C

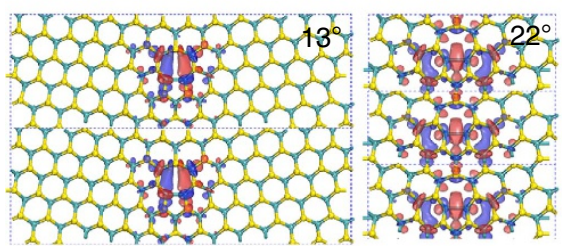

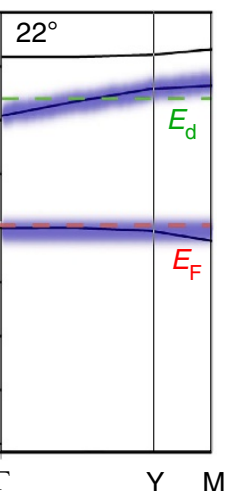

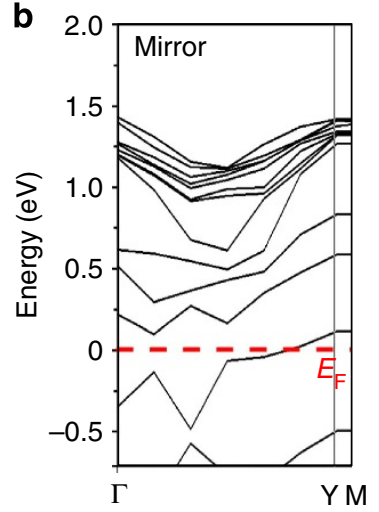

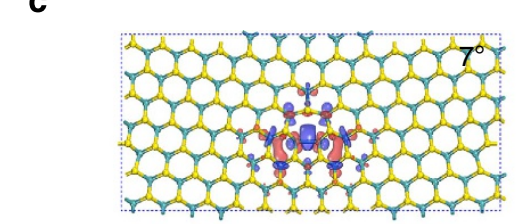

d $E_{\text {vacuum }}--$

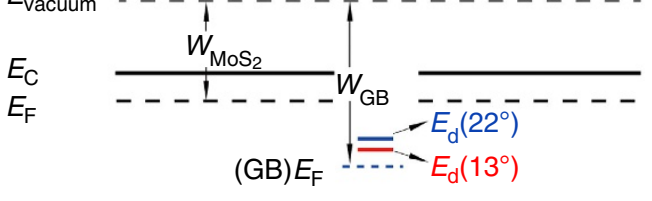

$E_{\mathrm{V}}$

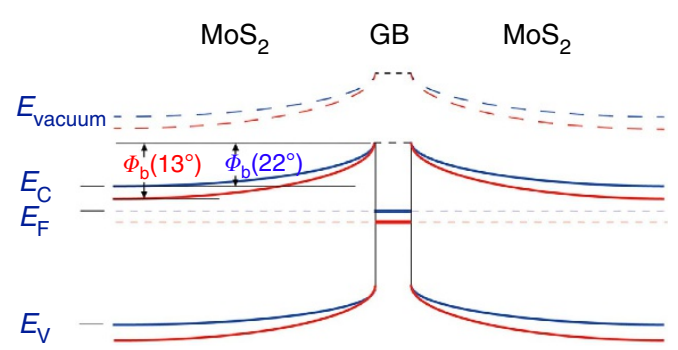

Figure 4 | DFT calculations of the band structure for GBs with various misorientation angles. Band structure near the Fermi level (red dash line) of (a) tilt and (b) mirror GBs. Inset shows the first Brillouin zone. The first band above the Fermi level is highlighted by a green-dashed line. (c) The charge densities for the first band above the Fermi level, as indicated by the 'Down' type of 5-7 dislocation cores for $7^{\circ}, 13^{\circ}$ and $22^{\circ}$. (d) The expected flat band alignment near the GB with defect states extracted from the band structures and the corresponding band bending after Fermi level equilibrium.

The Schottky barrier height decreases in response to $\alpha$.

C). The prepared $0.2 \mathrm{mM}$ AHM precursor was mixed with SC promoter in DI water and then spin-cast on $\mathrm{SiO}_{2} / \mathrm{Si}$ wafer. Prior to the spin-casting process, the solutions were mixed in the ratio $\mathrm{A}: \mathrm{B}: \mathrm{C}=0.55: 3: 1$. The mixing order $(\mathrm{A} \rightarrow \mathrm{B} \rightarrow$ handshake $\rightarrow C$ ) is critical to prevent micelle generation. The solution was dropped onto $\mathrm{SiO}_{2} / \mathrm{Si}$ wafer and followed by spin-casting at 2600 r.p.m. for $1 \mathrm{~min}$. A two-zone CVD system was used to control sulfur (zone 1) and substrate (zone 2) efficiently. Here, $200 \mathrm{mg}$ of sulfur (Sigma, 344621) was loaded into zone 1, while the substratecontaining metal precursor was placed at zone 2 . All growth in this work was carried out at atmospheric pressure. For growth, zone 1 was heated up to $210^{\circ} \mathrm{C}$ at a rate of $42^{\circ} \mathrm{C} \mathrm{min}^{-1}$. In the meantime, the temperature of zone 2 (growth temperature) were set to $780^{\circ} \mathrm{C}$.

Transfer of $\mathbf{M o S}_{\mathbf{2}}$ for device fabrication or TEM grid. Poly(methyl methacrylate) (PMMA A4, Chem) was spin-coated onto the as-grown $\mathrm{MoS}_{2}$ (2000 r.p.m., $1 \mathrm{~min}$ ) as a holder and protective layer with no post-annealing step. The $\mathrm{MoS}_{2}$ and PMMA support were then detached from the $\mathrm{SiO}_{2} / \mathrm{Si}$ substrate by floating the PMMA/ $\mathrm{MoS}_{2} / \mathrm{SiO}_{2} / \mathrm{Si}$, with the PMMA side up, in a hot 2-M NaOH solution. Samples grown on $\mathrm{SiO}_{2} / \mathrm{Si}$ were floated for less than $1 \mathrm{~min}$. Next, the PMMA/MoS 2 was washed by transferring the structure into DI water (4 times). Finally, the PMMA/ $\mathrm{MoS}_{2}$ was scooped out in pieces and placed on either a Quantifoil TEM grid with a gold-supported thin film (PELCO, 200 mesh, gold, 1.2- $\mu$ m holes) or a $\mathrm{SiO}_{2} / \mathrm{Si}$ wafer for device fabrication. The grids were stored under ambient conditions overnight to increase adhesion between the PMMA/MoS 2 and the grid. PMMA was ultimately removed by vapour acetone. The grids were then annealed at $180^{\circ} \mathrm{C}$ in a vacuum chamber at high pressure $\left(\sim 7.5 \times 10^{-5}\right.$ Torr $)$ for at least $12 \mathrm{~h}$ prior to TEM analysis. For device fabrication, the $\mathrm{MoS}_{2}$ was dried at $80^{\circ} \mathrm{C}$ for $5 \mathrm{~min}$. Before removal of the PMMA, the entire sample was immersed in DI water for a few seconds so as to wash away residual $\mathrm{NaOH}$ solution that remained on the surface of the PMMA during the detaching step. The sample was subsequently immersed in acetone for a few minutes to remove the PMMA, followed by cleaning in isopropyl alcohol.
Device fabrication. The synthesized $\mathrm{MoS}_{2}$ on an $\mathrm{SiO}_{2} / \mathrm{Si}$ substrate was transferred onto a highly doped $\left(10^{19} \mathrm{~cm}^{-3}\right)$ silicon substrate with a $100-\mathrm{nm}$-thick thermal oxide layer by a conventional transfer method mentioned in the previous TEM sample preparation. Glovebox preparation was employed for baking samples at $120^{\circ} \mathrm{C}$ for $\mathrm{SiO}_{2}$ and $\mathrm{MoS}_{2}$ surface trap passivation for $2 \mathrm{~h}$ before beginning fabrication. All further baking steps during fabrication were also performed in the glovebox. Electron beam lithography and evaporation (Ti/Au 7/35 nm) were used to define the $\mathrm{MoS}_{2}$ electrode contacts for each sample. $\mathrm{MoS}_{2}$ was patterned into an elongated Hall bar structure via a final electron beam lithography step, followed by a weak $10-\mathrm{W}$ plasma etch for $10 \mathrm{~s}$ under a $30-\mathrm{sccm}$ flow of $\mathrm{SF}_{6}$.

Transfer measured device to a TEM grid. Electrically characterized devices were transferred onto TEM grids to confirm the misorientation angle (SAED patterns) and the atomic structure of each GB (high-resolution STEM images). It is important to note that we succeeded in transferring the entirety of each device, which allowed for a direct investigation of the electrical behaviour and its correlation to both the atomic structure of the GBs and defects in $\mathrm{MoS}_{2}$. Here, the transfer process was similar to the one previously mentioned. However, the method employed in this study is compatible with $\mathrm{Ti} / \mathrm{Au}$ electrodes. While $\mathrm{Ti}$ is not the optimum contact for $\mathrm{MoS}_{2}$, it is more easily transferred, as the Ti layer is easy to detach from the $\mathrm{SiO}_{2} / \mathrm{Si}$ substrate compared to other metals such as $\mathrm{Cr}$. The transfer time for this process is longer than that associated with typical transfer procedures, which require less than a minute to detach the $\mathrm{PMMA} / \mathrm{MoS}_{2}$ from the $\mathrm{SiO}_{2} / \mathrm{Si}$ substrate.

TEM and DF-TEM. TEM imaging was conducted using a JEM ARM 200F machine operated at $80 \mathrm{kV}$; no damage was apparent in the samples at this accelerating voltage. The acquisition time for DF imaging was $1 \mathrm{~s}$ using the smallest objective lens aperture. The HR-TEM imaging acquisition time was also $1 \mathrm{~s}$. 
ADF-STEM. ADF-STEM imaging was conducted using a probe aberration-corrected JEM ARM200F operated at $80 \mathrm{kV}$. High-angle ADF images were acquired at $20 \mathrm{mrad}$ convergence angle. Because the energy of the low-voltage electron beam was below the damage threshold energy, the pristine $\mathrm{MoS}_{2}$ lattice remains stable and defect-free with regular scanning. Images presented in the main text were acquired with a medium-angle $\mathrm{ADF}$ detector with a collection angle ranging from 50 to $180 \mathrm{mrad}$ and an acquisition time of $40 \mu \mathrm{s}$ per pixel. We have used controlled imaging time (within $1 \mathrm{~min}$ ) under high-magnification STEM node to avoid beam damage on the $\mathrm{MoS}_{2}$ sample $6,24-26$.

Density functional calculations. DFT calculations performed with $\mathrm{DMOL}^{3}$ $\operatorname{code}^{27,28}$ were used to study the geometric structures and electronic states of different $\mathrm{MoS}_{2}$ monolayers. Geometries were fully optimized within a local spin density approximation with the Perdew - Wang correlational (LDA/PWC $)^{29}$ until the convergence criteria for energy, force and displacement were less than $10^{-5}$ Hartree, $4 \times 10^{-3}$ Hartree $\AA^{-1}$ and $5 \times 10^{-3} \AA$, respectively. All-electron basis sets were utilized for all elements. A Monkhorst-Pack sampling scheme with a $2 \times 2 \times 1 k$-point mesh was used for geometrical optimizations, and a $4 \times 8 \times 2$ $k$-point mesh was employed for electronic structure calculations. All models for structure optimization were supercells with a sufficiently large size and minimal defect interactions. The super sizes of all GB types were the same, as shown in Fig. 4c. For example, the supercell size is $43 \times 18 \times 20 \AA$ for the $7^{\circ} \mathrm{GBs}$.

\section{References}

1. Blatter, G. \& Greuter, F. Carrier transport through grain boundaries in semiconductors. Phys. Rev. B 33, 3952-3966 (1986).

2. Greuter, F. \& Blatter, G. Electrical properties of grain boundaries in polycrystalline compound semiconductors. Semicond. Sci. Technol. 5, 111-137 (1990).

3. Ando, T., Fowler, A. B. \& Stern, F. Electronic properties of two-dimensional systems. Rev. Mod. Phys. 54, 437-672 (1982).

4. Lu, H.-Z., Yao, W., Xiao, D. \& Shen, S.-Q. Intervalley scattering and localization behaviors of spin-valley coupled dirac fermions. Phys. Rev. Lett. 110, 016806 (2013)

5. Kang, K. et al. High-mobility three-atom-thick semiconducting films with wafer-scale homogeneity. Nature 520, 656-660 (2015).

6. Zhou, W. et al. Intrinsic structural defects in monolayer molybdenum disulfide. Nano Lett. 13, 2615-2622 (2013).

7. Zou, X., Liu, Y. \& Yakobson, B. I. Predicting dislocations and grain boundaries in two-dimensional metal-disulfides from the first principles. Nano Lett. 13, 253-258 (2013).

8. Najmaei, S. et al. Vapour phase growth and grain boundary structure of molybdenum disulphide atomic layers. Nat. Mater. 12, 754-759 (2013).

9. van der Zande, A. M. et al. Grains and grain boundaries in highly crystalline monolayer molybdenum disulphide. Nat. Mater. 12, 554-561 (2013).

10. Najmaei, S. et al. Electrical transport properties of polycrystalline monolayer molybdenum disulfide. ACS Nano 8, 7930-7937 (2014).

11. Sangwan, V. K. et al. Gate-tunable memristive phenomena mediated by grain boundaries in single-layer $\mathrm{MoS}_{2}$. Nat. Nanotechnol. 10, 403-406 (2015).

12. Zhu, W. et al. Electronic transport and device prospects of monolayer molybdenum disulphide grown by chemical vapour deposition. Nat. Commun. 5, 3087 (2014)

13. Cui, X. et al. Multi-terminal transport measurements of $\mathrm{MoS}_{2}$ using a van der Waals heterostructure device platform. Nat. Nanotechnol. 10, 534-540 (2015). 14. Salerno, J. P. et al. in Techn. Rep. Vol. 669 (MIT, 1984).

15. Jin Sheng, P. Fundamentals of Materials Science. 674 (Singhua University Press, 2011).

16. Lehtinen, O., Kurasch, S., Krasheninnikov, A. V. \& Kaiser, U. Atomic scale study of the life cycle of a dislocation in graphene from birth to annihilation. Nat. Commun. 4, 2098 (2013)
17. Ranganathan, S. On the geometry of coincidence-site lattices. Acta Crystallogr. 21, 197-199 (1966).

18. Humphreys, F. J. \& Hatherly, M. in Recrystallization and Related Annealing Phenomena 2nd edn 91-119 (Elsevier, 2004).

19. Jena, D., Gossard, A. C. \& Mishra, U. K. Dislocation scattering in a two-dimensional electron gas. Appl. Phys. Lett. 76, 1707-1709 (2000)

20. Look, D. C. \& Sizelove, J. R. Dislocation scattering in GaN. Phys. Rev. Lett. 82, 1237-1240 (1999).

21. Lu, C.-P. et al. States, and gating effects in $\mathrm{MoS}_{2}$. Nano Lett. 14, 4628-4633 (2014).

22. Jeffreys, $H$. On certain approximate solutions of linear differential equations of the second order. Proc. London Math. Soc. [2] 23, 428-436 (1925).

23. Huang, Y. L. et al. Bandgap tunability at single-layer molybdenum disulphide grain boundaries. Nat. Commun. 6, 6298 (2015).

24. Komsa, H.-P. et al. Two-dimensional transition metal dichalcogenides under electron irradiation: defect production and doping. Phys. Rev. Lett. 109, 035503 (2012).

25. Hong, J. et al. Exploring atomic defects in molybdenum disulphide monolayers. Nat. Commun. 6, 6293 (2015).

26. Azizi, A. et al. Dislocation motion and grain boundary migration in two-dimensional tungsten disulphide. Nat. Commun. 5, 4867 (2014).

27. Delley, B. From molecules to solids with the DMol3 approach. J. Chem. Phys. 113, 7756-7763 (2000)

28. Delley, B. An all-electron numerical method for solving the local density functional for polyatomic molecules. J. Chem. Phys. 92, 508-517 (1990).

29. Perdew, J. P. \& Wang, Y. Accurate and simple analytic representation of the electron-gas correlation energy. Phys. Rev. B 45, 13244-13249 (1992).

\section{Acknowledgements}

This work was supported by the Institute for Basic Science (IBS-R011-D1). Q.D. would like to thank the Deutsche Forschung Gemeinshaft (Project PO 1602/1-1). The authors also wish to thank Prof. You-hua Luo (East China University of Science and Technology), who provided the $\mathrm{DMOL}^{3}$ package.

\section{Author contributions}

T.H.L., D.J.P. and J.Z. contributed to this work in the experimental planning, experimental measurements, data analysis and manuscript preparation. Q.D. contributed to the theoretical calculations. H.K. and G.H.H. synthesized $\mathrm{MoS}_{2}$ on $\mathrm{SiO}_{2} / \mathrm{Si}$ wafer. S.H.C. and H.Y.J. aided with device fabrication. Y.H.L. contributed to the experimental planning, data analysis and manuscript preparation.

\section{Additional information}

Supplementary Information accompanies this paper at http://www.nature.com/ naturecommunications

Competing financial interests: The authors declare no competing financial interests.

Reprints and permission information is available online at http://npg.nature.com/ reprintsandpermissions/

How to cite this article: Ly, T. H. et al. Misorientation-angle-dependent electrical transport across molybdenum disulfide grain boundaries. Nat. Commun. 7:10426 doi: $10.1038 /$ ncomms10426 (2016).

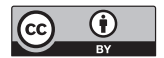

This work is licensed under a Creative Commons Attribution 4.0 International License. The images or other third party material in this article are included in the article's Creative Commons license, unless indicated otherwise in the credit line; if the material is not included under the Creative Commons license, users will need to obtain permission from the license holder to reproduce the material. To view a copy of this license, visit http://creativecommons.org/licenses/by/4.0/ 